\title{
Um Relato de Experiência do Acolhimento d@s Calour@s do Departamento de Ciência da Computação da Universidade de Brasília
}

\author{
Maristela Holanda ${ }^{1}$, Marcelo Mandelli ${ }^{1}$, Edison Ishikawa ${ }^{1}$, Dilma da Silva ${ }^{2}$ \\ ${ }^{1}$ Departamento de Ciência da Computação - Universidade de Brasilia (UnB) \\ Brasília - DF - Brasil \\ ${ }^{2}$ Department of Computer Science - Texas A\&M University \\ College Station - Texas - United States \\ \{mholanda, mgmandelli, ishikawa\}@unb.br, dilma@cse.tamu.edu
}

\begin{abstract}
The first year of an undergraduate major is a challenge for most firstyear students who enter the University of Brasilia. Of course, this challenge applies not only to them but to most first-year students at Brazilian and worldwide universities. This issue often leads the students to drop out of their majors. In this context, this paper presents the Program offered by the Department of Computer Science at the University of Brasilia which aims to welcome the new students to the major in a more friendly way. The program was carried out in its first edition in the first semester of 2020, and the lessons learned are presented.
\end{abstract}

\begin{abstract}
Resumo. O primeiro ano de um curso de graduação é um desafio para muitos calouros e calouras que ingressam na Universidade de Brasília. Esse desafio não é apenas local, mas está em diferentes universidades brasileiras e mundiais. A dificuldade no primeiro ano do curso leva, muitas vezes, o aluno a desistirem dos seus cursos. Neste contexto, este artigo apresenta o Programa de Acolhimentod@sCalour@sdo Departamento de Ciênciada Computaçãoda Universidade de Brasília com o objetivo de recepcionar de uma maneira mais amigável os alunos novos no curso. O programa foi realizado em sua primeira edição no primeiro semestre de 2020, e a sua estrutura, assim como também as lições apreendidas são apresentadas neste artigo.
\end{abstract}

\section{Introdução}

Como diminuir a evasão em cursos de graduação tem sido tema de pesquisa de diferentes universidades e instituições no mundo. Como pode ser observado no relatório da ACM (Association for Computing Machinery) em [Stephenson et al. 2018], nas instituições americanas, questões que se destacam em relação à retenção dos alunos em Computação envolvem: as dificuldades no primeiro ano do aluno em um curso superior em Computação; a primeira matéria de programação; assim como também a diferença de background e a questão da falta de diversidade entre os alunos de Computação. Outros estudos, também destacam como é complexo para o aluno de Computação o seu primeiro ano no curso [Prickett et al. 2020, Giannakos et al. 2017, Vesikivi et al. 2020].

Este cenário internacional não muda no Brasil [Marques et al. 2020]. Inclusive [Carvalho et al. 2019] observou uma forte associação entre a participação dos calouros 
nas atividades de acolhimento e a existência de ações afirmativas com o seu desempenho no primeiro período letivo do curso, o que indica a contribuição deste tipo de atividades na retenção dos calouros.

O Departamento de Computação (CIC) que oferece quatro cursos em nível de graduação, Ciência da Computação, Engenharia da Computação, Engenharia Mecatrônica, e Licenciatura em Computação, tem tido elevadas taxas de evasão. Por exemplo, a taxa de desistência no curso de Licenciatura em Computação (noturno) é aproximadamente $83 \%$ e no curso de Ciência da Computação (diurno) é de 65\% [CPA/UnB 2019]. Estas taxas são bem diferentes quando comparadas com outros cursos na UnB, como o de Engenharia Elétrica que a desistência é aproximadamente $20 \%$, e do curso noturno Engenharia de Produção, 38\%.

O Departamento de Computação da UnB tem tomado várias ações para diminuir essas taxas de evasão: reformulação do currículo, modificando as disciplinas do primeiro semestre dos cursos; reformulação da primeira disciplina de programa, Algoritmo e Programação de Computadores (APC) e, consequentemente, da segunda disciplina de programação (Estrutura de Dados); várias pesquisas sobre o perfil dos alunos que ingressam no curso; dentre outras ações.

Neste contexto, este relato de experiência apresenta uma das ações para melhorar o acolhimento dos novos alunos do CIC, o Programa de Acolhimento d@s Calour@s do Departamento de Computação executado no semestre de 2020.1, com o objetivo de motivar os alunos a persistirem nos seus cursos. Este programa foi inspirado em uma disciplina de seminários da Texas A\&M University com o nome Engineering Student Success Seminar que tem como objetivo recepcionar os alunos do primeiro ano em engenharia da universidade. Em algumas aulas são apresentadas oportunidades e recursos que os estudantes podem ter durante o seu periodo universitário na Texas A\&M University. É importante destacar que o CIC sempre fez um acolhimento dos seus calouros, porém o mesmo era realizado em uma recepção de um único encontro com uma ou duas horas de duração organizado pelas coordenações dos cursos de graduação. Outras universidade brasileiras também tem ações para a recepção de estudantes em cursos de Computação, porém a maioria é com a temática de inclusão de mulheres na Computação [Mochetti et al. 2016, Bordin et al. 2020, Siqueira 2020].

A primeira edição do Programa de Acolhimento d@s Calour@s do CIC, em particular, se reveste de uma característica muito peculiar devido ao seu início durante o período da pandemia da COVID-19. Além da necessidade dos calouros se adaptarem ao ritmo das atividades acadêmicas, eles tiveram que se adaptar ao ensino remoto (forma de ensino que não atende às normas exigidas para o ensino a distância, mas necessária para substituir as aulas presenciais de forma provisória e emergencial). Neste sentido, o desafio foi duplo, acolher os calouros e fazê-los sentir incluídos em um ambiente de isolamento social.

Este artigo está composto das seguintes seções: Seção 2 apresenta o perfil dos ingressantes no Departamento de Ciência da Computação da UnB; Seção 3, a metodologia, onde os oito encontros são detalhados e seus objetivos são apresentados; Seção 4 o perfil dos alunos que participaram do programa é apresentado; Seção 5 as avaliações realizadas pelos alunos de todos os encontros são descritas; Seção 6 as lições aprendidas 
são apresentadas; por fim, na Seção 7 tem as conclusões e os trabalhos futuros.

\section{Ingressantes no Departamento de Ciência da Computacão da UnB}

A Figura 1 gerada a partir de dados do sistema acadêmico da UnB apresenta a porcentagem dos alunos de escola pública que ingressaram nos cursos de graduação do CIC de 2009 até 2019, incluindo os cursos de Ciência da Computação, Licenciatura em Computação, Engenharia Mecatrônica e Engenharia da Computação. Como é possível observar antes de 2012, apenas em média, 20\% dos alunos que ingressavam no nosso departamento eram de escola pública. A partir da Lei $\mathrm{N}^{\mathrm{o}} 12.711$ sobre o ingresso em universidade federais [Brasil 2012] esse perfil mudou, e com a total implementação da cota em 2016, ingressam no departamento aproximadamente 50\% de alunos de escolas públicas.

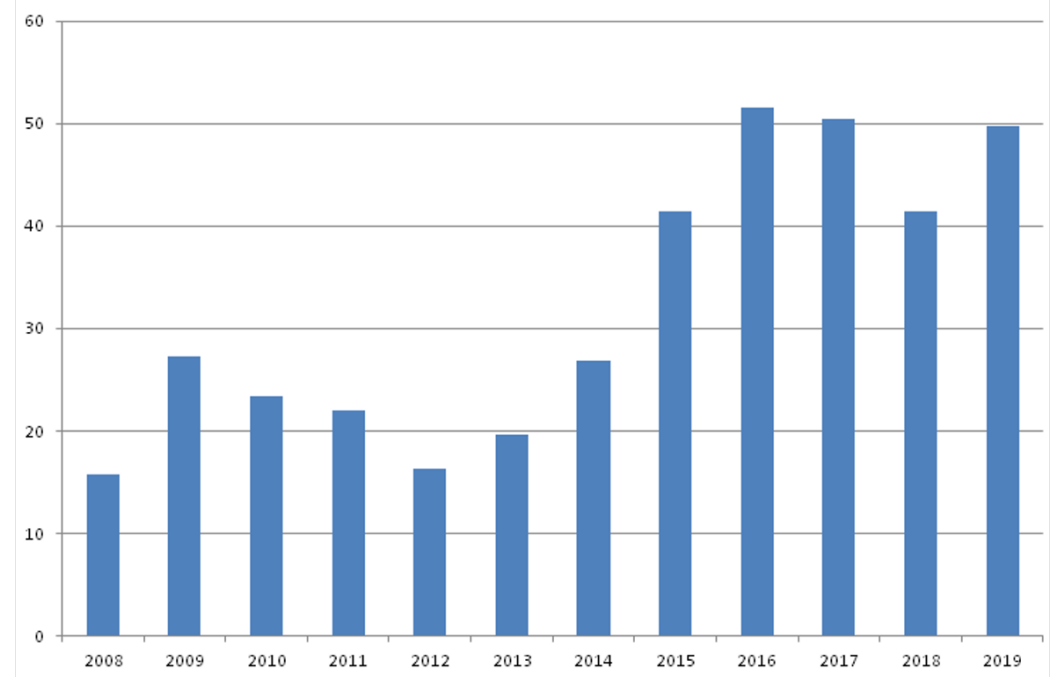

Figura 1. Porcentagem de Alunos de Escola Publica Ingressantes no CIC.

A UnB foi uma das primeiras universidades públicas a adotar o sistema de cotas, com as cotas raciais em 2003, e uma das primeiras a implementar a Lei $\mathrm{N}^{\mathrm{o}} 12.711$ sobre o ingresso em universidade federais [Brasil 2012]. Contudo, não basta apenas o sistema de cotas para o resgate social, mas também a fixação destes alunos no curso até a sua graduação.

O CIC em todos os seus cursos de graduação busca a excelência na formação de seus egressos, e como manter essa excelência, uma vez que os alunos das cotas tem que superar o gap educacional e socioeconômico em relação aos alunos da concorrência universal, tem sido um desafio que o departamento tem trabalhado com diferentes ações.

\section{Metodologia do Programa}

Durante o semestre de 2020.1, por causa da pandemia, a metodologia foi baseada em encontros virtuais realizados pela plataforma Teams da Microsoft, composta por palestras e momentos de interação com os alunos ingressantes no departamento. Foram oito encontros de 50 minutos, às quartas no horário de $18 \mathrm{~h}-18 \mathrm{~h} 50$. A escolha da realização do encontro do programa, no final da tarde, foi para possibilitar os alunos do curso do diurno 
Tabela 1. Encontros do Programa.

\begin{tabular}{|l|l|l|}
\hline No. & Encontro & Objetivo \\
\hline 1 & Abertura & Apresentação do programa e do departamento. \\
\hline 2 & Onde pedir apoio na UnB & Apresentação das possibilidades de apoio da UnB. \\
\hline 3 & Dicas de estudo e organização do tempo & Apresentação de técnicas de organização dos estudos. \\
\hline 4 & Projetos de Alunos para os alunos & Palestra dos alunos para os alunos. \\
\hline 5 & História de Professores do Departamento & Apresentar as dificuldades do primeiro ano do curso de graduação. \\
\hline 6 & História de ex-alun@ @ & Os ex-alunos devem apresentar o seu primeiro ano no departamento. \\
\hline 7 & Fala d@ @ Calour@s & Apresentação e Interação entre os calour@ @s. \\
\hline 8 & Encerramento & Fechamento do programa. \\
\hline
\end{tabular}

(Ciência da Computação, Engenharia da Computação e Engenharia Mecatrônica) e noturno (Licenciatura de Computação) participarem do evento sem terem choque de horário com suas disciplinas. A duração de 50 minutos, é que neste momento de pandemia, com atividades apenas online, a duração de menos de uma hora seria importante para manter a atenção dos alunos.

Um dos pilares do programa foi a diversidade de gênero. O CIC tem o projeto Meninas.comp que desde 2010 trabalha com a inclusão de mulheres em Computação [Holanda et al. 2016], sendo um programa colaborador do Meninas Digitais da Sociedade Brasileira da Computação (SBC). Sendo assim, em todos os encontros tivemos homens e mulheres, tentando manter uma equidade de gênero dos apresentadores. Na UnB, nos cursos de Computação, em média, ao longo dos últimos 10 anos, tivemos $15 \%$ de meninas nos cursos de Computação. Outro ponto importante foi a escolha de palestrantes que tiveram dificuldades em seu primeiro ano de curso. O objetivo é que os alunos ingressantes entendam que é normal essa dificuldade e mesmo assim eles tem potencial para terminarem os cursos e serem bons profisisonais.

A Tabela 1 apresenta os encontros e os seus respectivos objetivos. Como pode ser observado, cada dia teve um foco diferente, mas todos com a visão de ajudar o aluno a ter sucesso no seu curso e, principalmente, a motivarem os alunos a continuarem seus cursos de graduação.

No Encontro 1 o Departamento de Ciência da Computação foi apresentado para os calouros e calouras do curso. O encontro foi composto por: uma abertura de 5 minutos apresentando o Programa de Acolhimento d@s Calour@s, e em seguida os gestores do departamento falaram por 5 minutos cada. A gestão do curso é composta por: o Diretor do Instituto de Ciência Exatas, a Chefia (chefe e vice-chefe) do departamento, os coordenadores de cada curso do departamento e a secretaria acadêmica. Nesta ocasião foi dada a oportunidade dos funcionários da secretaria se apresentarem e se fazerem conhecidos dos alunos, tornando o atendimento menos impessoal, principalmente nesses tempos de isolamento social.

No Encontro 2 foram apresentados os programas de apoio ao discente da UnB. A UnB tem uma rede de apoio ao discente que vai desde apoio financeiro para alunos com necessidade financeira, apoio psicológico para os discentes, programas de extensão até iniciação científica entre os outros. Para esse encontro um professor do departamento apresentou durante 20 minutos e em seguida houve uma interação com os alunos.

No Encontro 3 uma professora do departamento apresentou técnicas de organização de estudos e de concentração. Este encontro foi baseado no material da 
aula Thinking about Thinking da Texas A\&M University, e do Centro de Excelência Acadêmica da Louisiana State University (https://www.lsu.edu/cas/). Foram 30 minutos de apresentação e depois perguntas dos alunos.

No Encontro 4, os alunos dos cursos de Computação em estágios mais avançados apresentaram para os calouros projetos que eles podem participar na UnB. Dentre as apresentações tiveram centros acadêmicos dos cursos de graduações, empresas juniores, projetos Meninas.comp e DROID (equipe de competição em robótica). Cada grupo teve 8 minutos para apresentar, e em seguida foi aberto para perguntas dos calouros.

No Encontro 5 foram apresentadas histórias de professores, com falas de uma professora e um professor do departamento. Escolhemos professores que tiveram dificuldade no primeiro semestre no curso de Computação, mas que se recuperaram e hoje são pesquisadores bem sucedidos.

No Encontro 6, a mesma ideia do encontro 5 foi aplicada para a escolha dos exalunos que apresentaram suas histórias, tendo o cuidado de também incluir ex-alunas do departamento que também tiveram dificuldades nos primeiro ano da universidade. Nesta atividade, procurou-se egressos com a faixa etária próxima a dos calouros e calouras para uma comunicação mais próxima dos calouros.

No Encontro 7 os alunos presentes se apresentaram, falaram em qual escola fizeram o ensino médio, explicaram o motivo da escolha pelo curso de Computação, e relataram como estava o primeiro semestre para eles. Neste encontro o objetivo era que os calouros conhecessem um pouco da história dos alunos que entraram com eles em Computação.

Por fim, no Encontro 8 foi o encerramento, um período de interação entre todos que participaram dos encontros, docentes e discentes, sugestões foram apresentadas pelos calouros e o que eles acharam do programa.

\section{Perfil dos Alunos e Alunas do Programa}

O programa teve a inscrição de 63 alunos, porém 19 participaram das atividades do programa e por fim concluíram o programa 13 alunos. A Figura 2 apresenta o perfil desses alunos. Na Figura 2a é apresentada a idade dos alunos. Como é possível observar a maioria está entre 18 e 20 anos que é a idade regular para ingressar em cursos de graduação na UnB.

$\mathrm{Na}$ Figura $2 \mathrm{~b}$ tem-se o perfil do gênero dos estudantes. Como esperado a maioria é composta por homens, totalizando 73,7\% (14 estudantes) dos participantes, e $25.3 \%$ de mulheres. Como um dos pilares do programa é a questão de diversidade de gênero esta informação é muito importante para o acompanhamento dessas calouras no curso. Como indicado anteriormente, o Meninas.comp durante os seus 11 anos de atuação em escolas públicas de ensino médio conseguiu incluir mulheres nos cursos de Computação da UnB. Porém, essas alunas tiveram muita dificuldades nos seus primeiros anos, sendo assim, precisava da continuidade em termos um ambiente mais woman friendly no CIC para mantê-las no curso.

Na Figura 2c, tem-se que a maioria dos calouros são cotistas (57.9\%), não cotista $(36.8 \%)$ e um aluno entrou pelo sistema de cota racial. Desta forma, o aluno que entrou 
pela vaga de Cota da UnB racial/indigena foi preenchida, cumprindo a função de resgate das minorias desfavorecidas pela universidade pública.

Por fim, na Figura 2d, tem-se a resposta da pergunta "Seu pai ou sua mãe fizeram curso superior", isto é, o aluno é a primeira geração da família a cursar ensino superior. Nesta figura é possível observar que mais de 50\% dos alunos que participaram do programa são a primeira geração da família, o que corrobora a necessidade de um acolhimento e uma orientação mais próxima destes alunos.

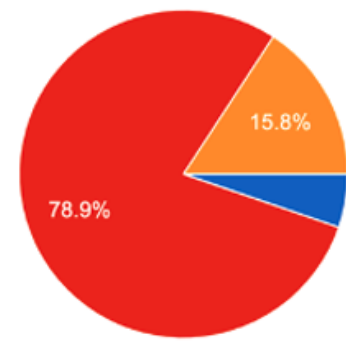

a) Idade. Vermelho (18 a 20 anos), Laranja (20 a 23 anos) e Azul (menos que 17)

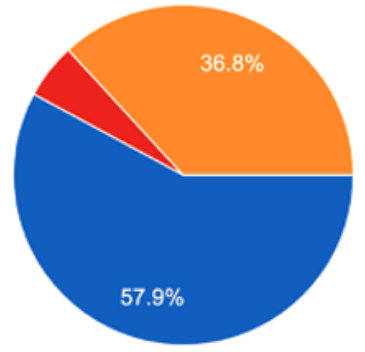

c) Entrou por cota. Azul (Sim, Cota Social) Vermelho (Cota Racial), Laranja (Não)

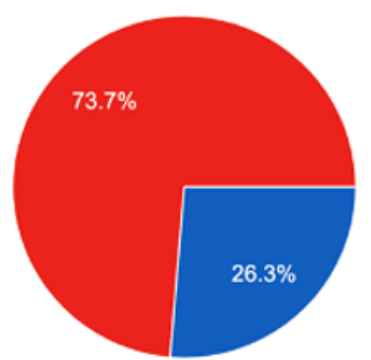

b) Gênero. Vermelho (masculino) e Azul (feminino)

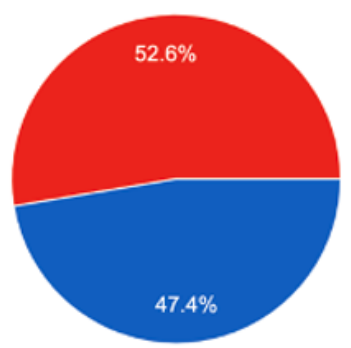

d) Seu pai ou sua mão tem curso superior. Azul (Sim) Vermelho (Não)

Figura 2. Perfil do Aluno que Participou do Programa.

\section{Avaliação dos Encontros}

A partir do segundo encontro houve uma avaliação das atividades e solicitação de comentários, com objetivo de ter a visão dos alunos sobre o encontro e de melhorarmos para as próximas edições. A seguir, são apresentadas as avaliações dos encontros. Todos os encontros tiveram avaliações positivas que variaram dentre $76.9 \%$ até $97 \%$ quando perguntado aos discentes se o encontro foi útil.

O Encontro 2 "Onde pedir apoio na UnB" foi avaliado positivamente pelos calouros, sendo que aproximadamente $89.5 \%$ (17 estudantes) responderam que foi útil o encontro. Apenas dois alunos reportaram que "não foi útil pois já conhecia tudo que foi apresentado". Nesta atividade a maioria dos comentários foi elogiando a apresentação.

O Encontro 3 "Dicas de estudo e organização do tempo" foi avaliado positivamente pelos calouros, sendo que aproximadamente 76.9\% (10 estudantes) responderam que foi útil o encontro. Nesta atividade solicitamos que os alunos comentassem o motivo 
da escolha de Computação. Alguns comentários selecionados são: "Pobreza e vontade de evoluir", "Disposição de estudar não dependendo da minha escola e professores" e a "Dedicação e vontade de participar de algo que considero muito bom e importante para a minha formação". Em relação à parte da gestão do tempo, a maioria dos comentários foram positivos e esse solicitou mais detalhe sobre a metodologia adaptada de [Christ 1981], "Apresentar de forma mais aprofundada o método de estudo utilizado, achei muito bacana esse processo e pretendo pratica-lo, mas me senti na vontade de aprender mais sobre".

O Encontro 4 "Projetos de Alunos para os alunos" foi avaliado positivamente pelos calouros, sendo que aproximadamente 92.2\% (13 estudantes) responderam que foi útil o encontro. Apenas um aluno falou que foi mais ou menos útil. Os comentários foram positivos para esse encontro, sendo que um dos alunos escreveu: "Mais informações de como participar dos projetos".

O Encontro 5 "História de Professores do Departamento" foi avaliado positivamente pelos calouros, sendo que aproximadamente $88.9 \%$ (8 estudantes) responderam que foi útil o encontro. Os comentários foram positivos para esse encontro, sendo que um dos alunos escreveu: "achei interessante o quanto eles ressaltaram que cada aluno tem seu tempo/método de aprender e a importância do estudo em grupo", "Aproveite as oportunidades", e "A parte de sair da zona de conforto". Neste encontro também perguntamos se eles acham interessante conhecer um pouco mais sobre a vida dos professores, e todos os alunos responderam que sim.

O Encontro 6 "História de ex-alun@s" foi avaliado positivamente pelos calouros, sendo que aproximadamente 91\% (11 estudantes) responderam que foi útil o encontro. Este encontro foi o que teve maior número de perguntas pelos calouros e uma maior interação. Os comentários foram positivos para esse encontro, sendo que um dos alunos escreveu: "A área da computação, tem muitas oportunidades de emprego, dentro e fora do Brasil.", "As escolhas que eles tiveram que tomar ao longo da carreira acadêmica e profissional ", "Saber que reprovar pode ser algo comum", "A experiência dos convidados.", "Algumas dicas de estudos e de organização eu achei bem valiosas ", "Quando eles falaram sobre nao tinha certeza sobre o curso e querer desistir" e "Que eles são alunos de computação licenciatura".

O Encontro 7 "Fala d@s Calour@s”foi avaliado positivamente pelos calouros, sendo que aproximadamente $85.7 \%$ (12 estudantes) apontaram que é interessante se apresentar no Programa de Acolhimento d@s Calour@s. Os comentários foram positivos para esse encontro, sendo que um dos alunos escreveu: "Conhecimento de outras experiências é sempre enriquecedor.", "Muito bacana compartilhar as histórias e apresentações, pois a gente se sente mais seguro no curso em ter uma prévia do que vai acontecer e essa interação é muito importante ainda mais nesse momento de pandemia".

Por fim, no Encontro 8, os alunos fizeram uma avaliação geral sobre o Programa de Acolhimento d@s Calour@s do CIC. A avaliação geral foi positiva, sendo que 78.6\% (11 estudantes) dos alunos responderam que foi útil o programa, é interessante ter esse programa.

Como sugestões, as principais foram: Criar um canal do Discord para os calouros; Falar um pouco mais sobre cada curso do departamento; e iniciar mais cedo o programa de acolhimento. Um comentário dos alunos que destacamos é: "Trabalhar mais didáticas 
de métodos de estudo para os alunos, e chamar mais ex - alunos para falar um pouco de suas trajetórias e experiências, achei esse tipo de encontro o melhor de todos. Acredito que ambas as coisas que eu citei motivam muito os alunos, pelo menos me motivaram".

Com o intuito de acompanharmos a efetividade da participação dos alunos no Programa de Acolhimento, no início do semestre de 2020.2 (Março de 2021) foi solicitado que os alunos respondessem um formulário de percepção. Foram recebidas 12 respostas, das quais tem-se:

- Você acha que foi útil para o seu primeiro semestre na universidade ter participado do Programa de Acolhimento d@s Calour@s do CIC? Todos os alunos responderam que SIM;

- Você indicaria esse Programa para@s calour@s desse semestre? Todos os alunos responderam que SIM;

- Após a participação no programa você ficou mais motivado a continuar no seu curso? 75\% dos alunos (9) responderam SIM, e 25\% (3) responderam TALVEZ.

Como pode ser observado com as respostas, os alunos acreditam que o programa colaborou no sentindo de motivá-los a persisterem nos seus cursos de Computação. Não foi recebebida nenhuma resposta negativa (NÃO) para as perguntas do formulário.

\section{Lições Aprendidas}

Este período foi difícil pois a pandemia interrompeu as atividades na universidade em março de 2020, após apenas duas semanas de aulas, retornando de modo remoto apenas em setembro, com isso o programa só iniciou em outubro. De qualquer forma, destacamos algumas lições aprendidas:

- Dificuldade no engajamento dos alunos, porém esse período de pandemia não é possível tirarmos conclusões, uma vez que muitos alunos estão esperando a volta presencial para iniciar os estudos na UnB;

- Convidar mais alunos e ex-alunos para os encontros;

- Em todos os encontros ter fala de mais de um docente;

- Funcionou bem o horário, uma vez que tivemos alunos de Ciência da Computação e Engenharia Mecatrônica (diurno) e Licenciatura em Computação (noturno);

- Conseguimos oficializar o programa no departamento, assim os alunos podem solicitar carga horária de atividade complementar;

- Iniciar o programa na segunda semana de aula do semestre letivo;

- Criação de um canal do discord;

- Continuaremos com a questão de equidade de gênero, em todos os encontros teremos homens e mulheres apresentando em todos os dias do programa, também estamos conseguindo patrocínio para termos camisetas e brindes para sorteio durante o encontro.

O Programa de Acolhimento d@s Calour@s do CIC está em sua segunda edição, iniciado em março de 2021, onde já incluimos algumas questões apresentada nas lições aprendidas, como por exemplo:

- Criação do canal no discord;

- Divulgação do evento em todos os canais de comunicação do departamento, redes sociais, site do curso, lista de email dos calouros; 
- Adicionamos mais uma fala de aluno no Encontro 3 "Dicas de estudo e organização do tempo \& Apresentação de técnicas de organização dos estudos". Adicionalmente, incluimos neste encontro, uma parte de ferramentas para auxiliar na gestão do tempo neste período de sistema remoto, como o Trello e Google Agenda.

Essa segunda edição teve 60 inscrições, sendo que temos uma frequência média de 29 alunos nos 3 encontros iniciais. Para o encerramento, vamos fazer um campeonato de jogo eletrônico.

\section{Conclusões}

A evasão é um problema que impacta fortemente na formação dos alunos, e traz um prejuízo para todos os envolvidos no ensino público superior. O Departamento de Ciência da Computação da Universidade de Brasília tem realizado várias ações para melhorar os seus números. Dentre essas ações, destacam-se a reformulação da primeira disciplina de programação do curso, mudança da ordem de disciplinas no currículo, e com objetivo de motivar os alunos o Programa de Acolhimento d@s Calour@s.

O Programa de Acolhimento d@s Calour@s do CIC está em sua segunda edição, tivemos um aumento no número de participantes de 13 para em média 29 estudantes (alunos e alunas) da primeira para a segunda edição. De acordo com o nosso acompanhamento, as avaliações são bem positivas, mas ainda continuamos com o problema de engajamento dos alunos. De todo modo, neste período de pandemia, fica dificil concluir algo em relação ao engajamento.

Como trabalho futuro, pretende-se acompanhar os alunos que participaram das edições do programa de acolhimento do CIC e analisar a efetividade do programa na decisão dos alunos permanecerem nos seus cursos.

\section{Agradecimentos}

Agradecemos a Universidade de Brasíla pelo apoio ao Projeto CODIFICO: Programa para Tod@s Edital DPI/DPG 03/2020 - Apoio à execução de projetos de pesquisas científicas, tecnológicas e inovação. Assim como também, a Dra. Andrea Ogilvie e Dra. Elissa Morris, da Faculdade de Engenharia da Texas A\&M University, pelo material que elas prepararam para a disciplina CLEN 181 (lecionada pela co-autora Dilma Da Silva no Segundo semestre de 2020).

\section{Referências}

Bordin, A., Finger, A., Gindri, L., and de Mello, A. (2020). Tutoria das gurias: Uma ação de acompanhamento de alunas ingressantes em cursos de computação. In Anais do XIV Women in Information Technology, pages 129-138, Porto Alegre, RS, Brasil. SBC.

Brasil (2012). Lei No 12.711. http://portal.mec.gov.br/cotas/docs/ 1ei_12711_29_08_2012.pdf.

Carvalho, L., Santos, A., Nakamura, F., and Oliveira, E. (2019). Detecção precoce de evasão em cursos de graduação presencial em computação: um estudo preliminar. In Anais do XXVII Workshop sobre Educação em Computação, pages 233-243, Porto Alegre, RS, Brasil. SBC. 
Christ, F. L. (1981). Seven steps to better management of your study time. Personal Efficiency Programs.

CPA/UnB (2019). Relatório de perfil dos estudantes do departamento de ciência da computação (cic).

Giannakos, M. N., Pappas, I. O., Jaccheri, L., and Sampson, D. G. (2017). Understanding student retention in computer science education:the role of environment, gains, barriers and usefulness. Education and Information Technologies, 22(5):2365-2382.

Holanda, M., de Araújo, A. P., Walter, M. E., and de Oliveira, C. A. (2016). Meninas.comp: Um relato da experiência de integração entre o ensino médio e a universidade de brasília. In Anais do X Women in Information Technology, pages 78-82, Porto Alegre, RS, Brasil. SBC.

Marques, L. T., Marques, B. T., Rocha, R. S., Silva, L. C., Castro, A. F., and Queiroz, P. G. G. (2020). Evasão acadêmica e suas causas em cursos de bacharelado em ciência da computação: Um estudo de caso na ufersa. In Anais DO XXXI Simpósio Brasileiro de Informática na Educação (SBIE 2020), page 1042-1051.

Mochetti, K., Salgado, L., Zerbinato, A., Souza, B., and Avelino, M. (2016). Ciência da computação também é coisa de menina! In Anais do X Women in Information Technology, pages 11-15, Porto Alegre, RS, Brasil. SBC.

Prickett, T., Walters, J., Yang, L., Harvey, M., and Crick, T. (2020). Resilience and effective learning in first-year undergraduate computer science. In Proceedings of the 2020 ACM Conference on Innovation and Technology in Computer Science Education, page $19-25$.

Siqueira, T. (2020). Elas: introdução à engenharia da computação e além. In Anais do XIV Women in Information Technology, pages 20-29, Porto Alegre, RS, Brasil. SBC.

Stephenson, C., Miller, A. D., Alvarado, C., Barker, L., Barr, V., Camp, T., Frieze, C., Lewis, C., Mindell, E. C., Limbird, L., Richardson, D., Sahami, M., Villa, E., Walker, H., and Zweben, S. (2018). Retention in Computer Science Undergraduate Programs in the U.S.: Data Challenges and Promising Interventions. ACM, New York, NY, USA.

Vesikivi, P., Lakkala, M., Holvikivi, J., and Muukkonen, H. (2020). The impact of projectbased learning curriculum on first-year retention, study experiences, and knowledge work competence. Research Papers in Education, 35(1):64-81. 